\title{
Architecture Design of 2-D Discrete Wavelet Transformation Algorithm using Field Programmable Gate Array (FPGA)
}

\author{
Maha A. Hasso Sahla A. Ali \\ College of Computer Science and Mathematics \\ University of Mosul, Mosul, Iraq
}

Received on: 06/03/2013

Accepted on: 24/06/2013

\begin{abstract}
In this paper an architecture has been proposed for the 2-D Discrete Wavelet Transform (DWT) and the Inverse Discrete Wavelet Transform (IDWT) based on the Convolution method of the Daubechies 5/3-tap Biorthogonal filter bank in the Algorithm transform to image processing, and implementation it on the FPGA (Field Programmable Gate Array) using VHDL, for benefiting from implementation advantages of these Hardware and save run-time. The processing results proved speed and Efficiency of the proposed architecture, where the employed number of slices is less. So it result to Frequency higher and less run-time.The type of the FPGA based in this paper is Xilinx XC3S500E Spartan-3E using Xilinx ISE 9.2i.

Keywords: 2-D Discrete Wavelet Transformation, Field Programmable Gate Array (FPGA).

تصميم معمارية لخوارزمية تحويل المويجة المتقطع ذي البعدين باعتماد مصفوفة البوابات الحقلية القابلة

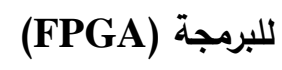

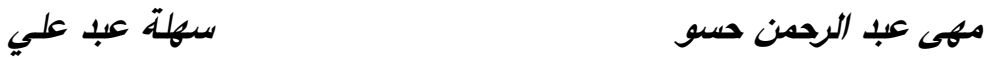

$$
\begin{aligned}
& \text { قسم علوم الحاسوب } \\
& \text { كلية علوم الحاسوب والرياضيات } \\
& \text { جامعة الدوصل }
\end{aligned}
$$

201316124 : تاريخ قبول البحث

$2013|3| 6$ : تاريخ استلام البحث
\end{abstract}

\begin{abstract}
الملخص
تم في هذا العمل اقتراح معمارية لخوارزمية تحويل المويجة المتقطع ذي البعدين (DWT) ومعكوس تحويل

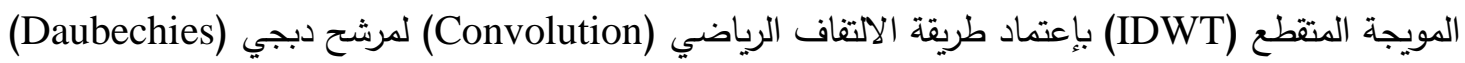
5/3 ثائي التعامد في خوارزمية التحويل لمعالجة الصورة وتطبيقه على مصفوفة البوابات الحقلية القابلة للبرمجة Field Programmable Gate Array (FPGA) من محاسن تطبيق هذه الاجهزة المادية وتوفير وقت التتفيذ. اثبتت النتائج العملية للتطبيق سرعة وكفاءة المعمارية

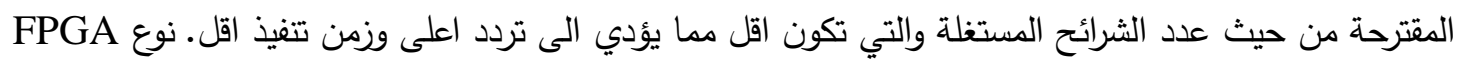

المتمد في هذا العمل هو Xilinx XC3S500E Spartan-3E وباستخدام برنامج الكلمات المفتاحية: تحويل المويجة المتقطع ذي البعدين، مصفوفة البوابات الحقلية القابلة للبرمجة.
\end{abstract}


1. المقدمة

تم اعتماد تحويلات المويجة لكبس الصورة والذي اصبح سائداً وبشكل واضح اكثر من تحويل جيب التمام

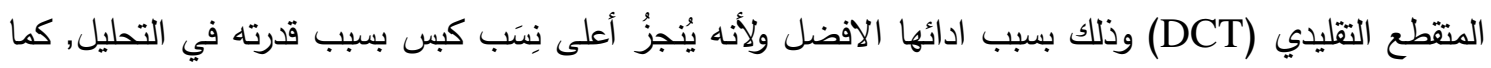

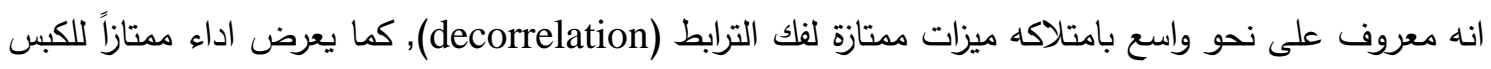
بفقدان ودون فقدان. ان الاداء العالي لخوارزمية 2D-DWT في كبس الصورة يُيرر استعماله نواةً في المعيار كPEG2000 لكبس الصور الثابتة [1] والمعيار MPEG-4 texture coding لكبس الصور المتحركة [2]. كما ان التحويل المويجي معروف على نحو واسع بامتلاكه ميزات ممتازة لفك الترابط, وهو يعرض اداءً ممتازاً

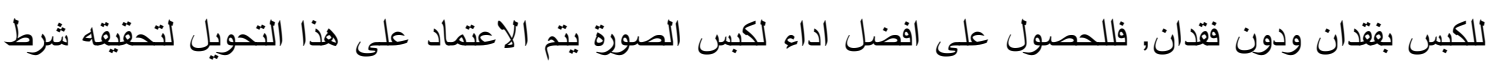

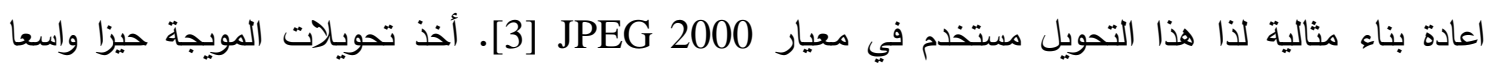
باهتمام الكثير من الباحثين وذلك لملاعمتها لكثير من الظواهر ولمختلف المجالات منها معالجة الاشارة والصورة,

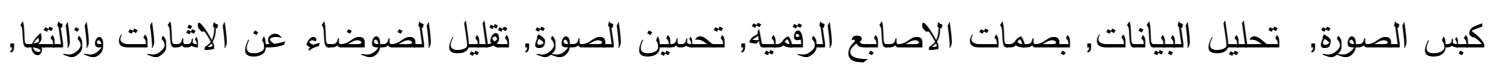
وتحليل إثارات الزلازل,... الخ[4]. وبسبب تزايد عدد التطبيقات في مناطق مختلفة, اصبح من الضروري استخدام

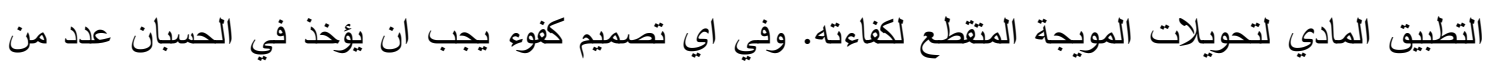

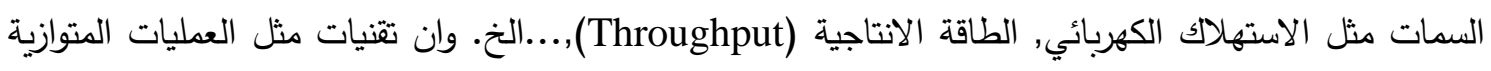
(Parallel Operations)

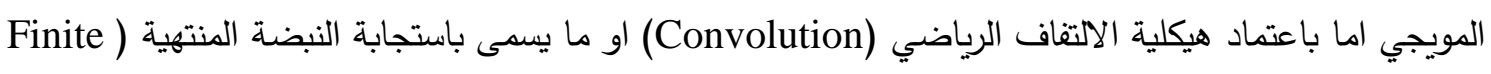
Impulse Response FIR مخطط الرفع (Lifting Scheme) للمويجة. تم اقتراح العديد من المعماريات لتحويل المويجة في السنوات السابقة]7], ففي المعمارية المقترحة في[8] دمجت التحويل بفقدان ودون فقدان , وفي [9] المعمارية المقترحة خفضت بثكل ملحوظ عدد الضوارب (multiplier) (الجوامع (adder) والسجلات اضافةً الى كمية الوصول للذاكرة الخارجية وأدت الى تقليل كلفة الجهاز واستهلاك القدرة بشكل كفوء في التصديم. 2. خوارزمية تحويل المويجة المتقطع ذي البعدين

يختلف تطبيق خوارزمية تحويل المويجة المتقطع ذات بعدين في VHDL في بعض السمات عن تطبيقه

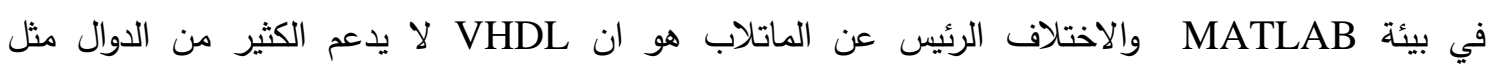

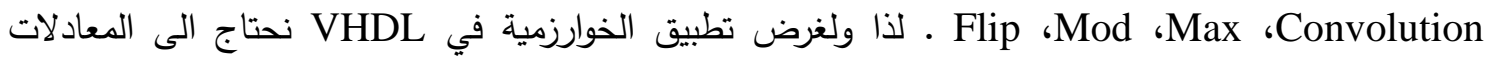
الخطية لتحويلات المويجة المتقطع ذات البعدين. تم في المعماريات المقترحة اعتماد المعادلات الحسابية لخوارزمية

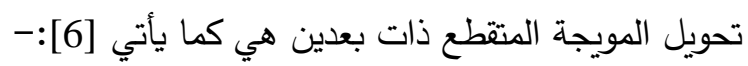
(1) $X_{L L}^{J}\left(n_{1}, n_{2}\right)=\sum_{i_{1}=0}^{k-1} \sum_{i_{2}=0}^{k-1} g\left(i_{1}\right) \cdot g\left(i_{2}\right) \cdot X_{L L}^{J-1}\left(2 n_{1}-i_{1}\right)\left(2 n_{2}-i_{2}\right)$ (2) $X_{L H}^{J}\left(n_{1}, n_{2}\right)=\sum_{i_{1=0}-1}^{k-1} \sum_{i_{2}=0}^{k-1} g\left(i_{1}\right) \cdot h\left(i_{2}\right) \cdot X_{L L}^{J-1}\left(2 n_{1}-i_{1}\right)\left(2 n_{2}-i_{2}\right)$ (3) $\mathrm{X}_{\mathrm{HL}}^{\mathrm{J}}\left(\mathrm{n}_{1}, \mathrm{n}_{2}\right)=\sum_{\mathrm{i}_{1=0}}^{\mathrm{k}-1} \sum_{\mathrm{i}_{2}=0}^{\mathrm{k}-1} \mathrm{~h}\left(\mathrm{i}_{1}\right) \cdot g\left(\mathrm{i}_{2}\right) \cdot \mathrm{X}_{\mathrm{LL}}^{\mathrm{J}-1}\left(2 \mathrm{n}_{1}-\mathrm{i}_{1}\right)\left(2 \mathrm{n}_{2}-\mathrm{i}_{2}\right)$ (4) $\mathrm{X}_{\mathrm{HH}}^{\mathrm{J}}\left(\mathrm{n}_{1}, \mathrm{n}_{2}\right)=\sum_{\mathrm{i}_{1=0}-1}^{\mathrm{k}-1} \sum_{\mathrm{i}_{2}=0}^{\mathrm{k}-1} \mathrm{~h}\left(\mathrm{i}_{1}\right) \cdot \mathrm{h}\left(\mathrm{i}_{2}\right) \cdot \mathrm{X}_{\mathrm{LL}}^{\mathrm{J}-1}\left(2 \mathrm{n}_{1}-\mathrm{i}_{1}\right)\left(2 \mathrm{n}_{2}-\mathrm{i}_{2}\right)$ حيث ان: 


$$
\begin{aligned}
& \text { تمثل مستوى تحويل المويجة المتقطع ذات البعدين. J } \\
& \text { K }
\end{aligned}
$$

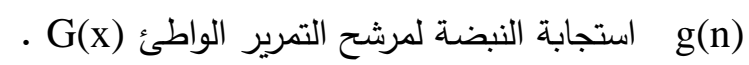

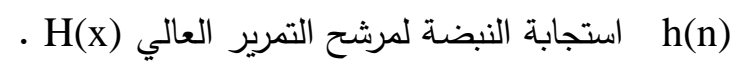

$$
\begin{aligned}
& \text { تمثل الصورة المدخلة. XLL }{ }^{0}\left(\mathrm{n}_{1}, \mathrm{n}_{2}\right)
\end{aligned}
$$

ان كل مستوى تحليل في DWT يتكون من مرحلتين, وكما مبين في الثكل(1): المرحلة الاولى تمرير

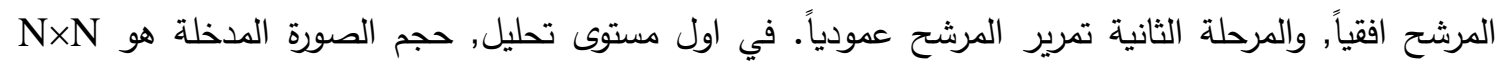

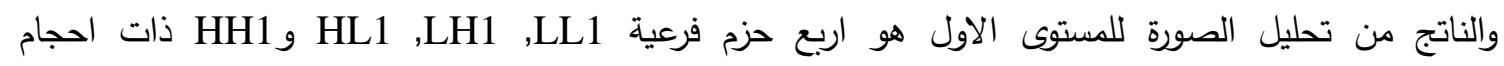

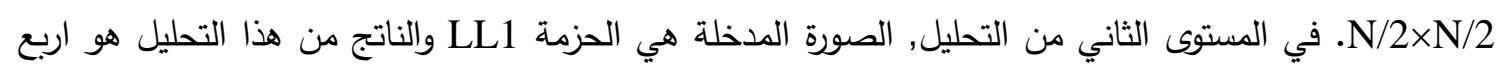

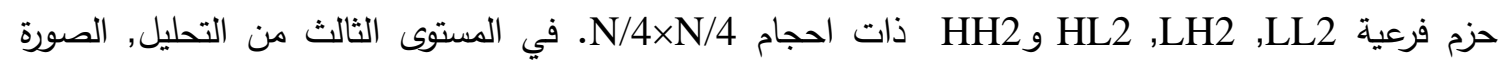

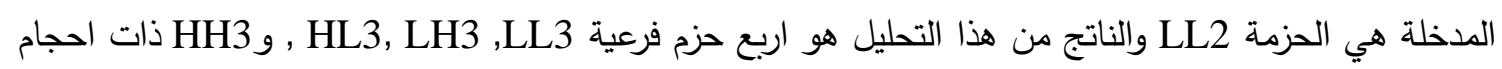
N/8×N/8. بنس الاسلوب تم الاستمرار في عملية تحليل الصورة, حجم الحزم الناتجة من التحليل يمكن حسابه التحابه

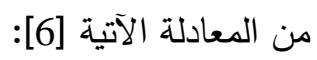

$$
\text { (5) } \sum_{\mathrm{L}=1}^{\mathrm{J}} \frac{\mathrm{N}}{2^{\mathrm{L}-1}}=\mathrm{N}+\frac{\mathrm{N}}{2}+\frac{\mathrm{N}}{2^{2}}+\frac{\mathrm{N}}{2^{3}}+\cdots+\mathrm{N} / 2^{\mathrm{J}-1}
$$$$
=2\left(1-2^{-\mathrm{J}}\right) \mathrm{N}
$$

$$
\text { حيث تمثل }
$$

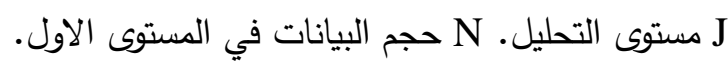

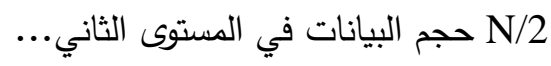

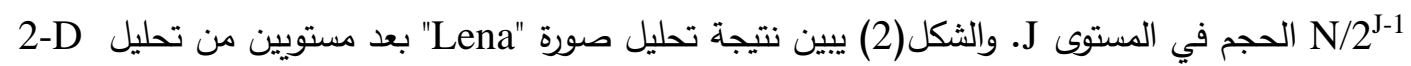




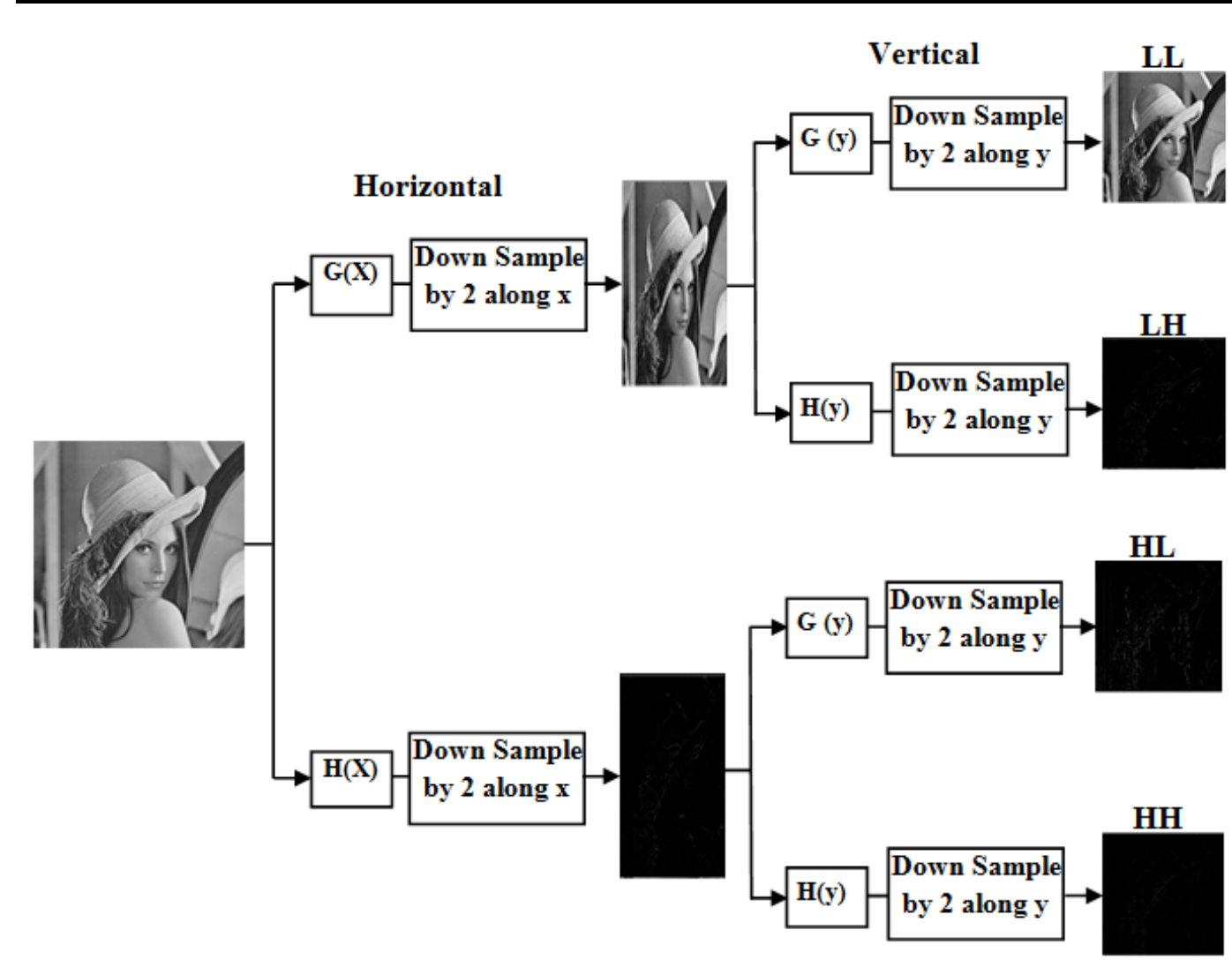

الثكل(1) تحليل 2-D DWT لمستوى واحد.
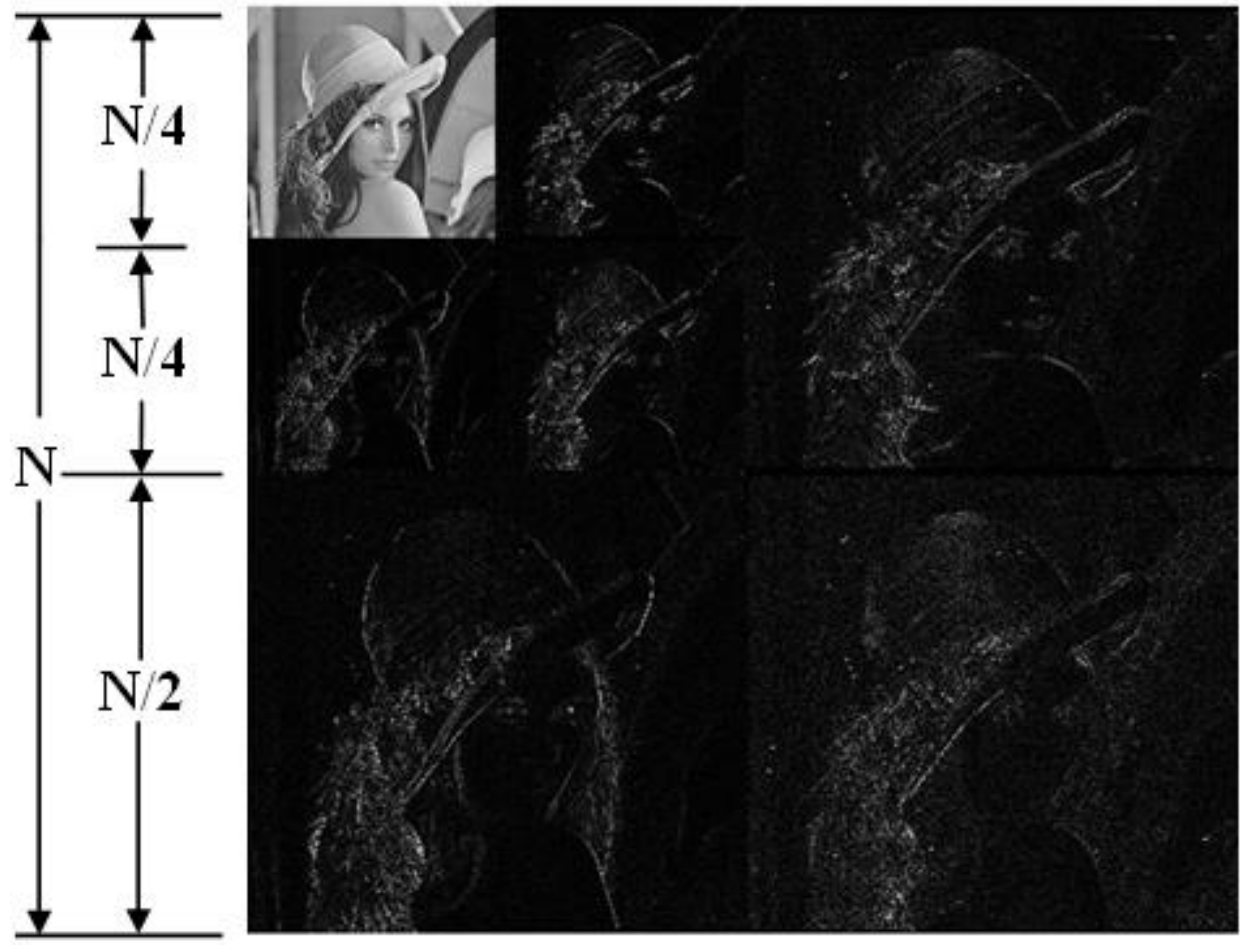

الثكل(2) نتيجة تحليل صورة "Lena" بعد مستويين من التحليل.

3. المعمارية المقترحة لخوارزمية 2D DWT-IDWT باعتماد معمارية الازاحة لتطبيق الاتفاف الرياضي: 
i : التطبيق العملي للمعمارية المقترحة لـ 2D DWT الأمامية لمستوى واحد

يختلف التعامل مع الصورة في لغة وصف الكيان المادي عنه في لغات اخرى اذ انه لا لامكنه قراءة الصورة بصيغتها القياسية مباشرةً من الحاسوب لذا يجب تغيير صيغة الصورة وتحويلها الى صيغة تفهمها لغة فئة فئه وصف الكيان المادي من هذه الصيخ: السداسي عشر (Hex-Format) او الثنائي (Binary-Format). لذا عند فئد

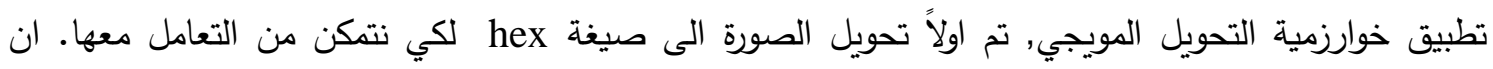

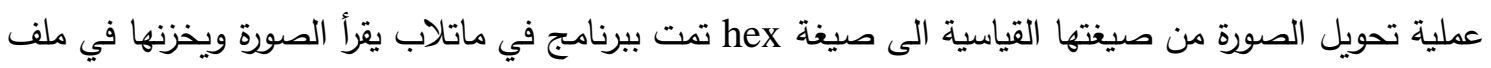

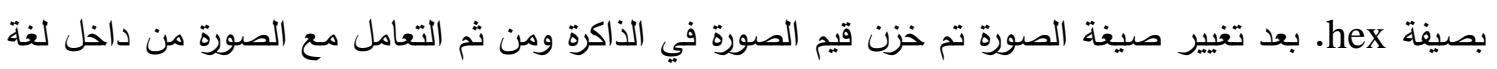
وصف الكيان المادي باستخدام برنامج ISE9.2i والثكل(3) يوضح التطبيق المادي للمعمارية المقترحة لخوارزمية التحويل المويجي المتقطع لمستوى واحد. كما مبين من الثكل ان الذاكرة التي أُستخدمت في المعمارية المقترحة

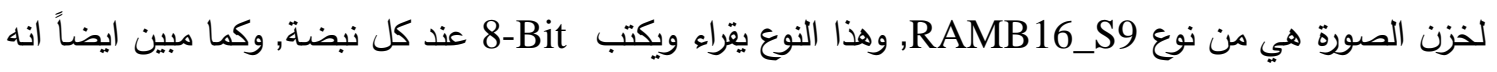

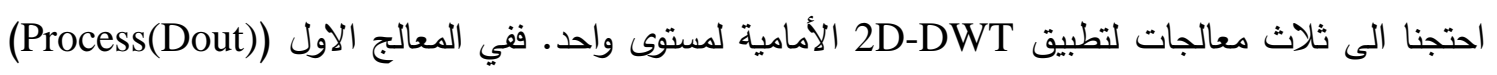

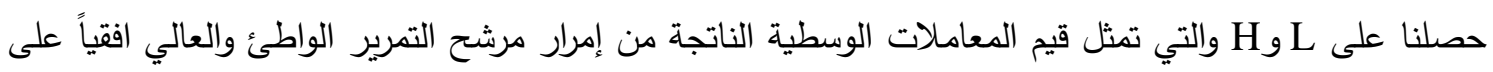
الصورة اما عند المعالجين (Process (H) (H) Process (L) حصلنا على HL,LH,LL و

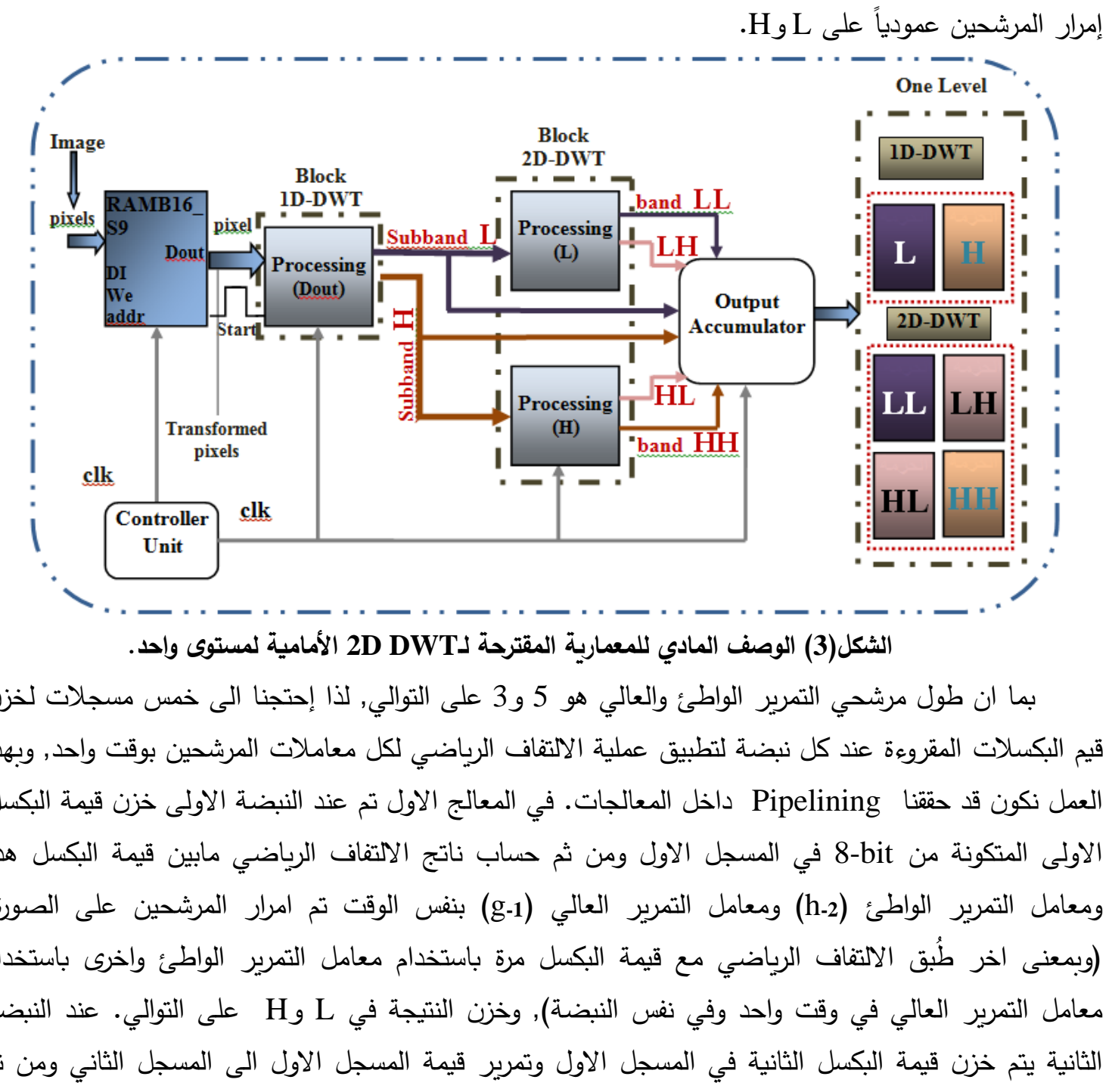


حساب ناتج الالتفاف الرياضي ما بين قيم المسجلين ومعاملي مرشح التمرير الواطئ (h-2,h-1) ومعاملي مرشح

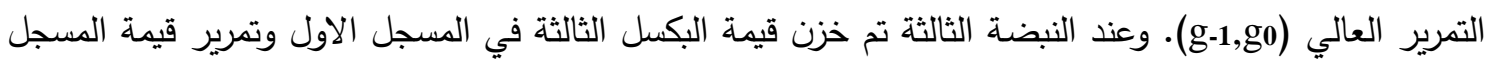

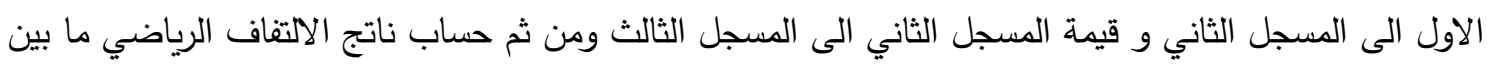

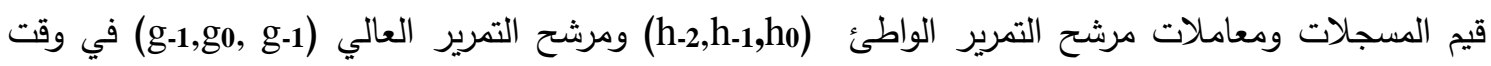
واحد وخزن النتيجة في L و H على التوالي, وهكذا تم تطبيق العمل نفسه على كل صفوف الصورة. تم ايضاً في

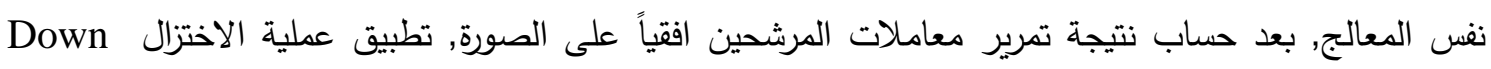

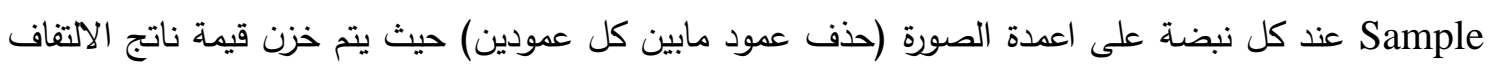

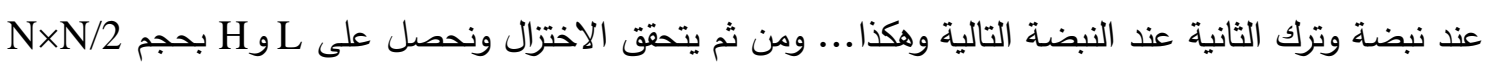

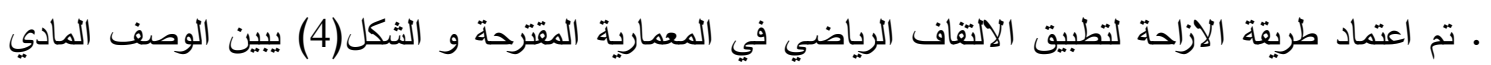

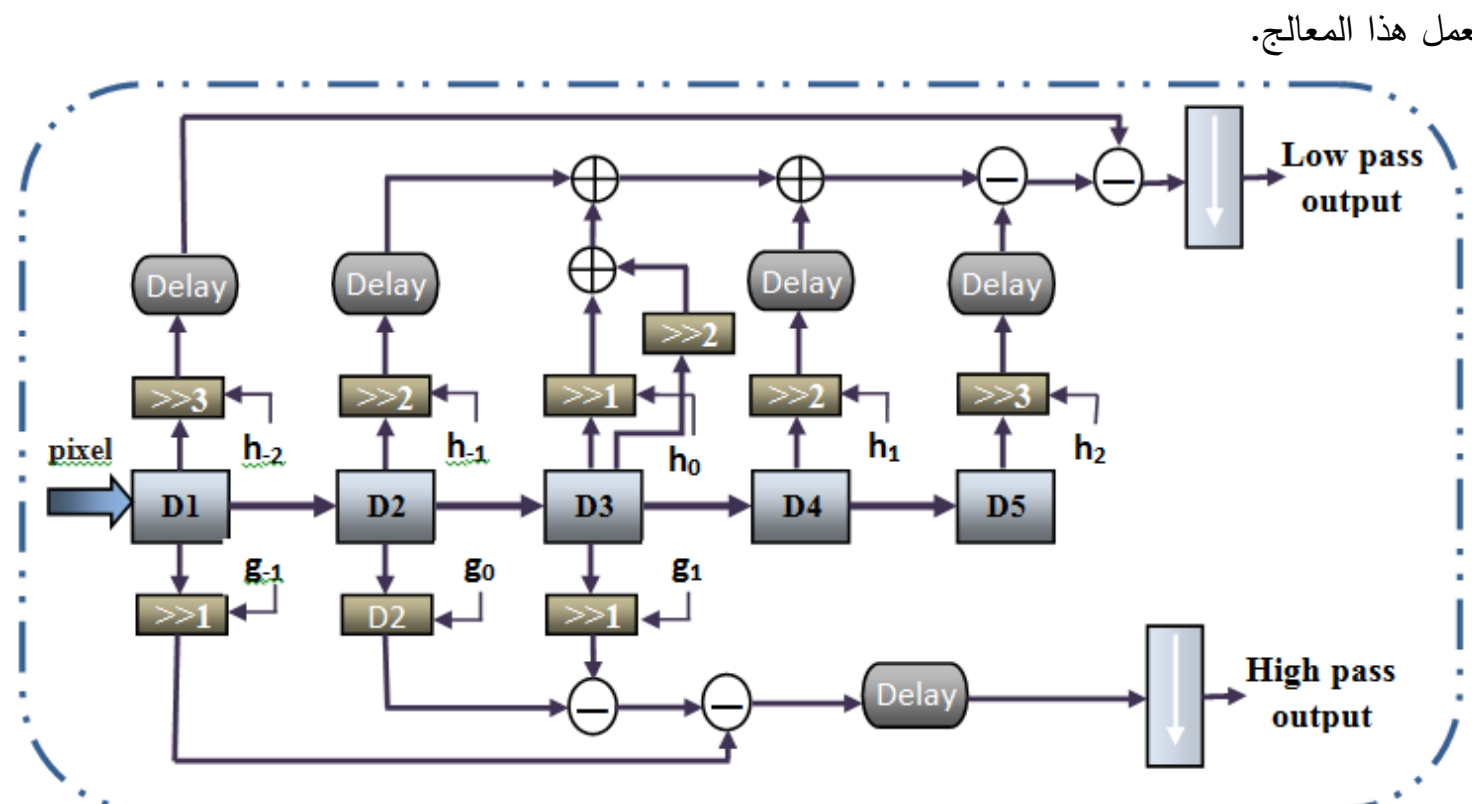

الثكل(4) الوصف المادي لعمل المعالج الاول (Process(Dout) •

$$
\text { حيث تمثل: }
$$

1>1 > > الازاحة الى اليمين بمقدار واحد, حيث ان هذه الازاحة تمثل عملية القسمة لقيمة البكسل على قيمة

المعامل 2. - ال

2> > الازاحة الى اليمين بمقدار اثنان, وهي تمثل عملية القسمة لقيمة البكسل على قيمة المعامل 4.

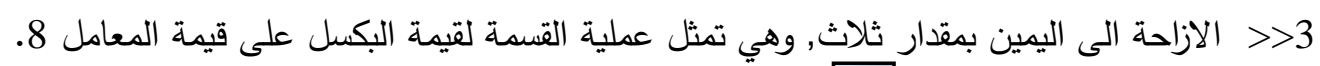
Delay

$$
\begin{array}{r}
\text { (h-2,h-1,ho,h1,h2) } \\
\text { معاملات مرشح التمرير الواطئل مرشح التمرير العالي. (g-1,g0,g1,) }
\end{array}
$$

كما مبين من الثكل, تم اضافة تأخير بمقدار نبضة واحدة على التصميم بسبب معامل مرشح التمرير

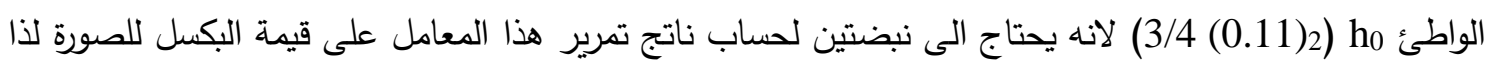


تم تأخير حساب قيم الالتفاف لباقي معاملات المرشحات بمقدار نبضة واحدة للحصول علي كل نتائج تمرير المعاملات على قيم البكسلات بنس الوقت وبنفس النبضة وبذلك تكون النتائج صحيحة ودقيقة. ان اعتماد طريقة الازاحة الى اليمين لتطبيق الالتفاف الرياضي في المعمارية المتترحة والتي تمثل عملية

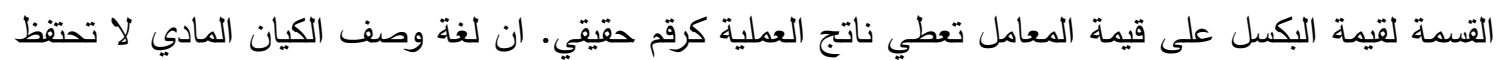

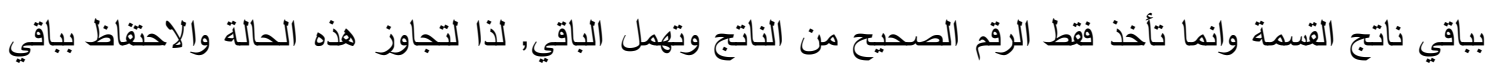

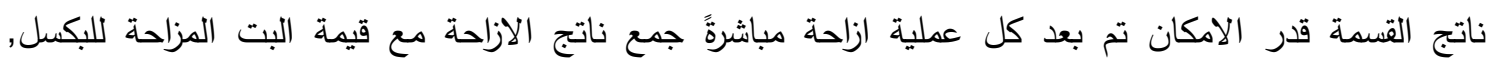

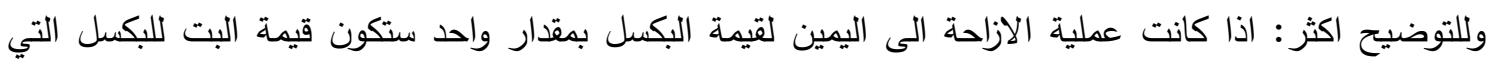

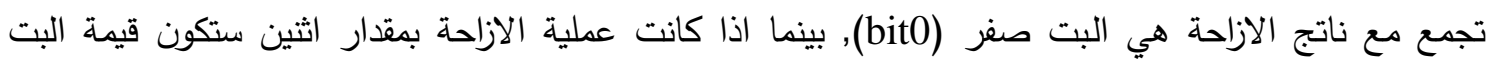

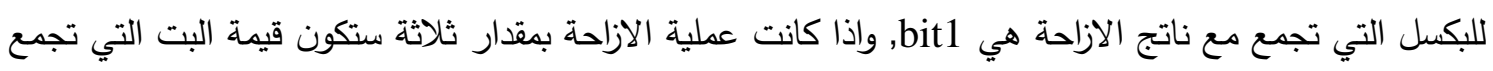

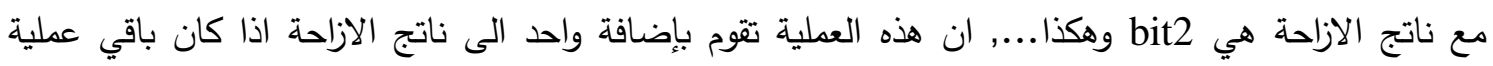

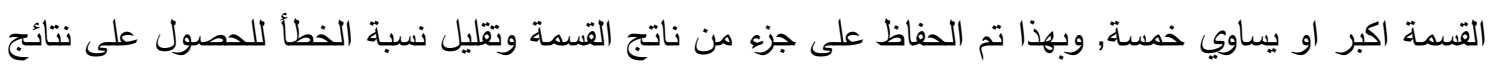

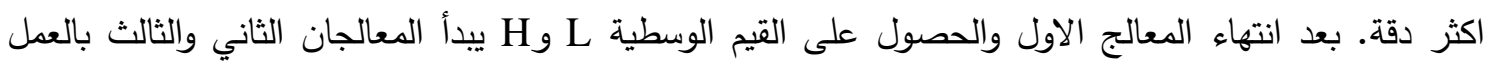

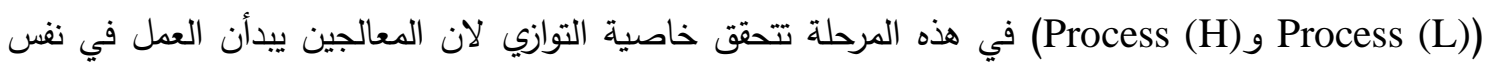

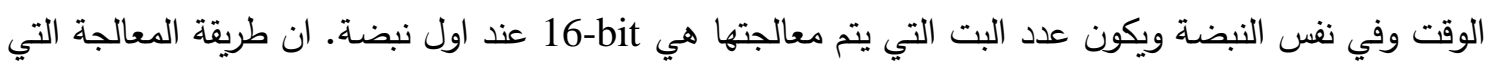

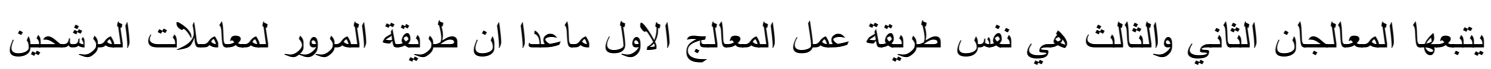

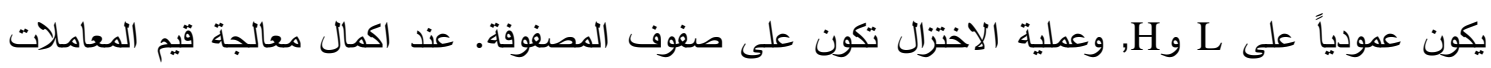

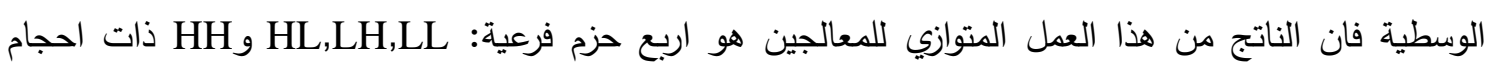

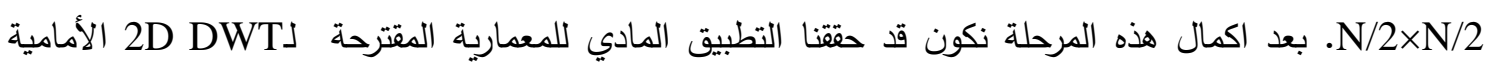
لكستوى واحد.

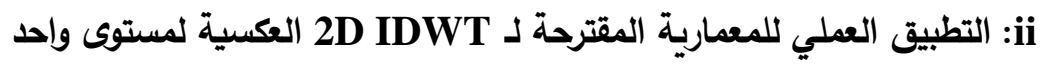

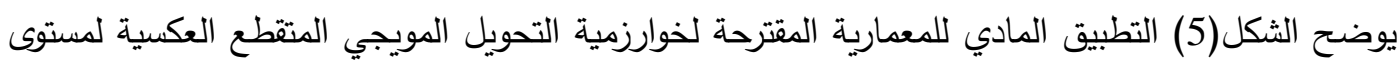
واحد. كما مبين من الثكل انه احتجنا الى ثلاث معالجات للمعمارية المتترحة العكسية لمستوى واحد, وكما ذكرنا

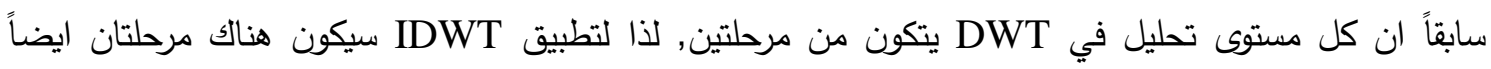

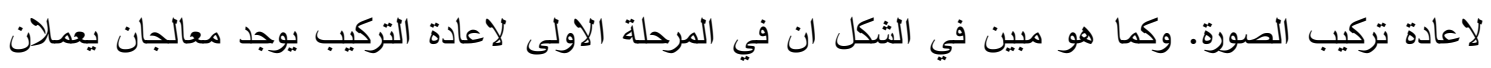

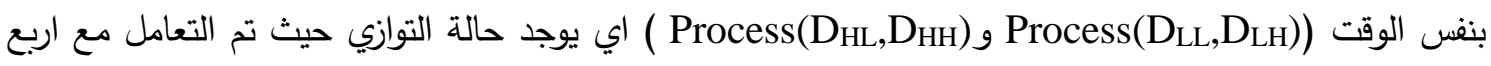
ذاكرات في وقت واحد عند كل نبضة, وبذلك يكون عدد البكسلات التي تعالج عند النبضة الاولى هي 4 بكسل

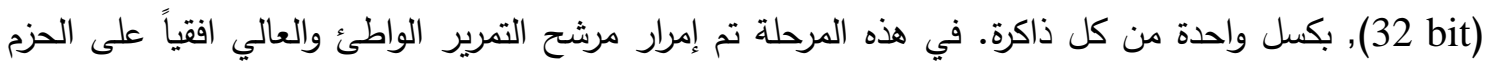

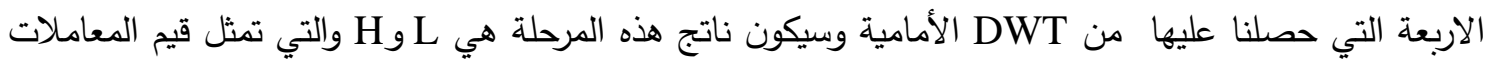

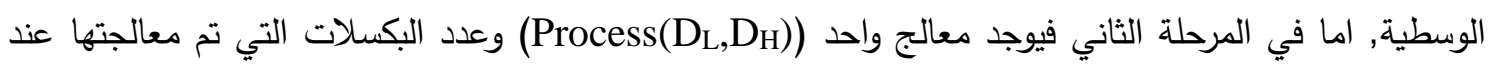

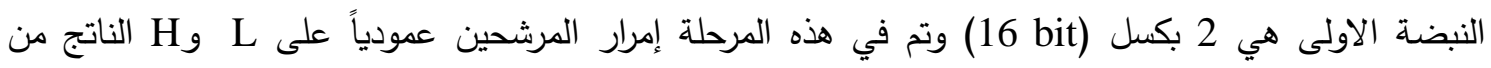
المرحلة الاولى, ناتج هذه المرحلة هي الصورة التي تم تركيبها من ناتج المرحلتين. 


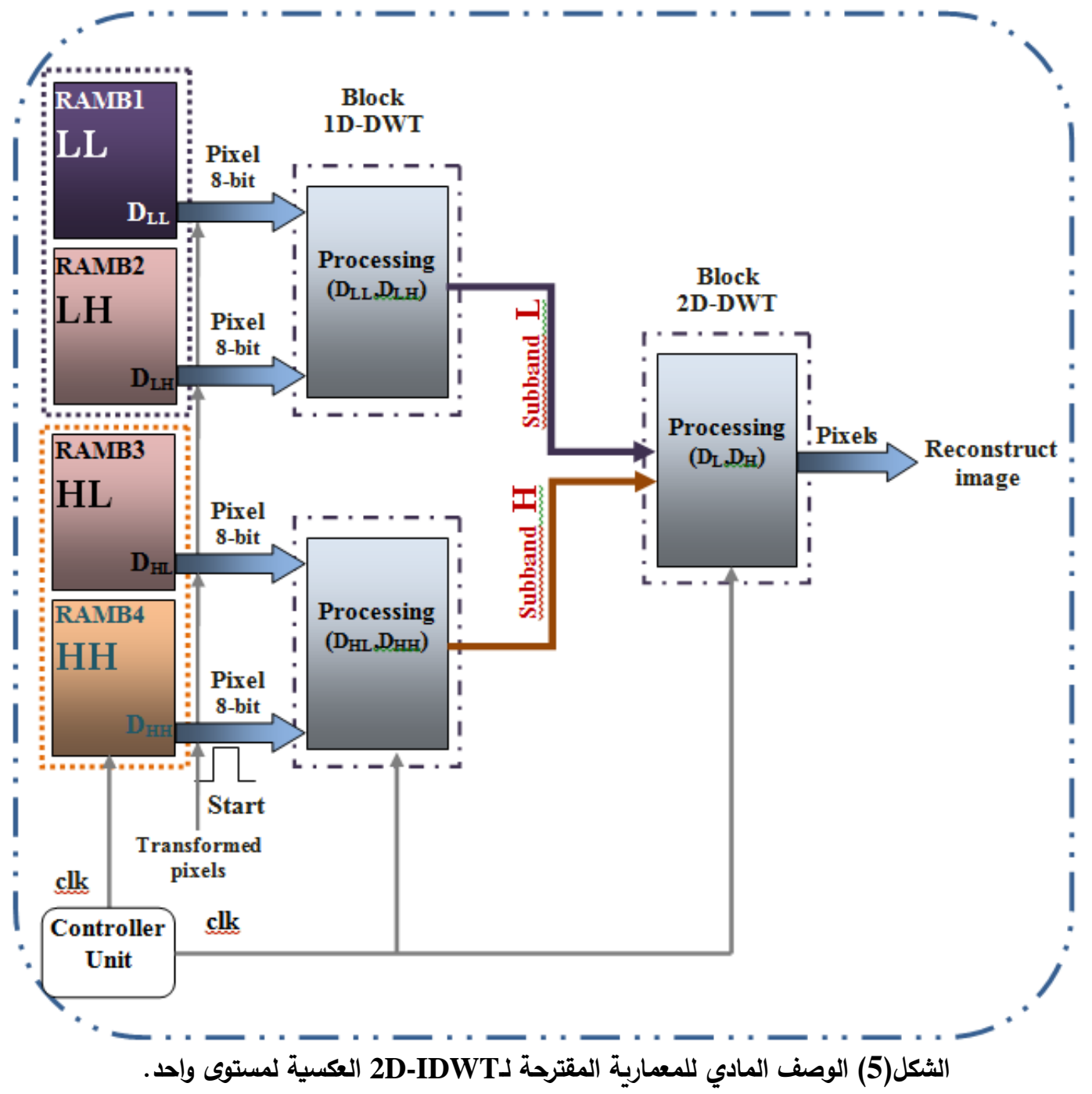

ان طريقة المعالجة المتبعة في هذه المعمارية هي كما يأتي: في المعالج الاول (Process(DLL,D نحتاج الى ثمان مسجلات لخزن قيم البكسلات المقروهة عند كل نبضة من الذاكرتين RAMB1 و

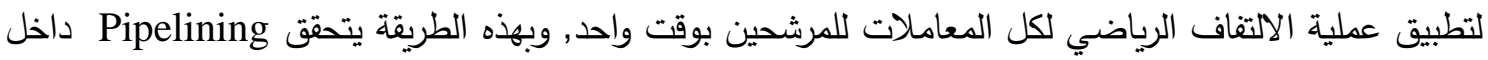
المعالج. ان الثكل (6) يبين بوضوح طريقة عمل هذا المعالج, كما يبين ايضاً انه تم استخدام ثلاث مسجلات لخزن قيم البكسلات المقروة من الذاكرة RAMB1 وخمسة مسجلات لخزن قيم البكسلات المقروءة من الذاكرة

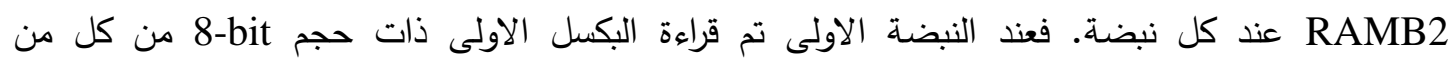
وخزنها في مسجلين, وتم بعدها حساب ناتج الالتفاف الرياضي مابين قيمة البكسل لذاكرة

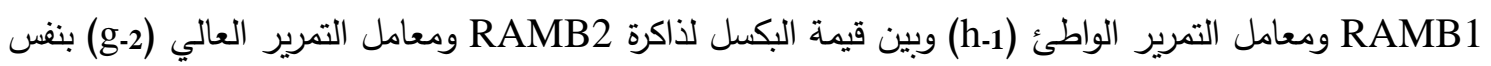

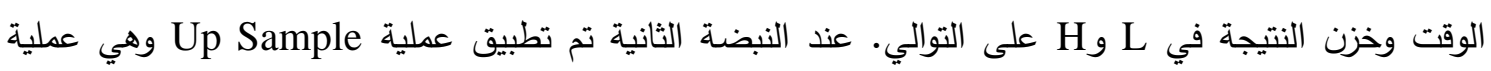
اضافة عمود صفري, حيث تم خزن القيمة صفر في المسجل الاول التابع لكل من RAMB1 و و RAMB2 وتمرير قيم المسجلين الى المسجل الثاني التابع لكل من الذاكرتين, ومن ثم حساب ناتج الالتفاف الرياضي ما ما بين

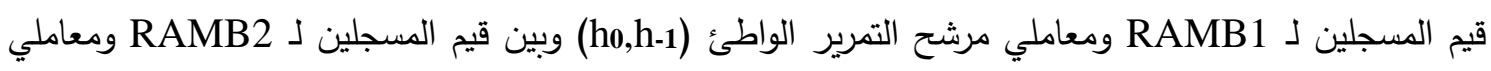


مرشح التمرير العالي (g-1,g0) وخزن النتيجة في L L H Hلى التوالي. وعند النبضة الثالثة تم خزن قيمة البكسل

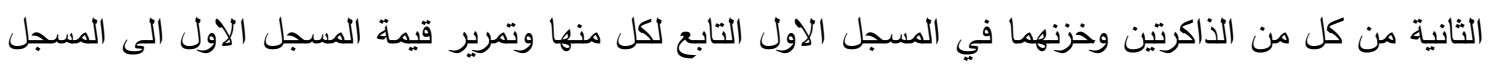

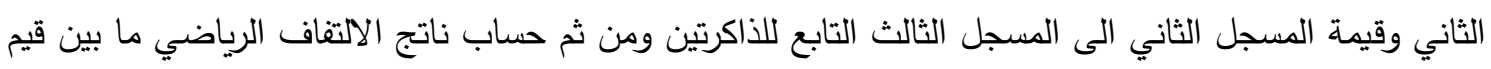

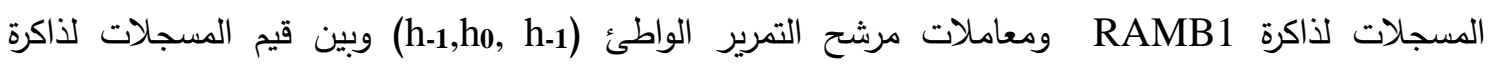
RAMB2

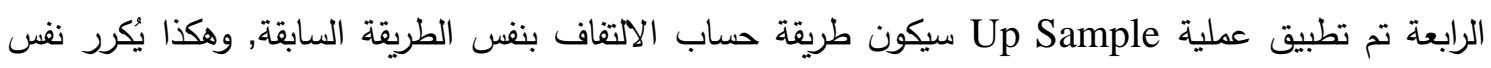

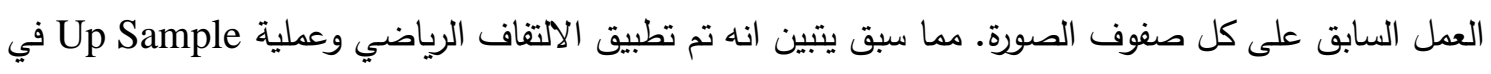

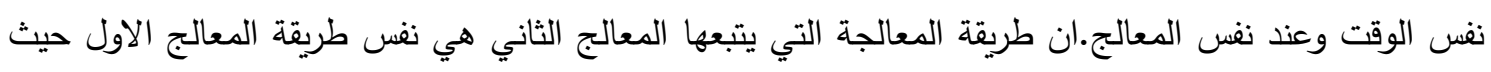

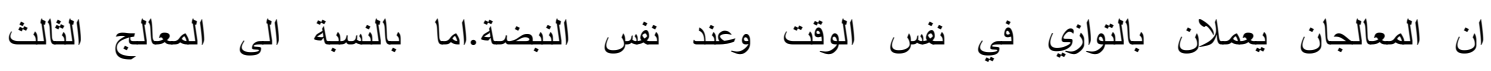
(Process(DL, DH

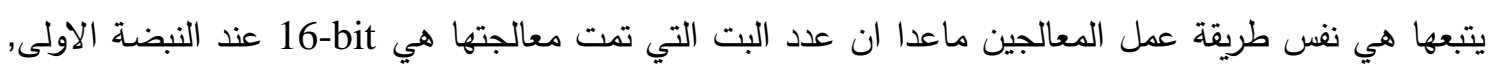

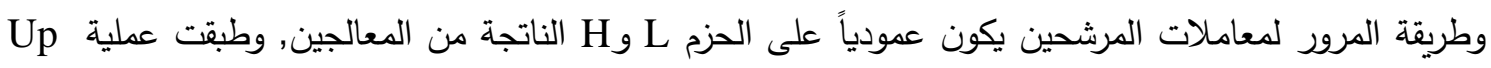
على الاعمدة أي تم اضافة عمود صفري. عند اكمال عملية المعالجة لقيم المعاملات الوسطية يتم

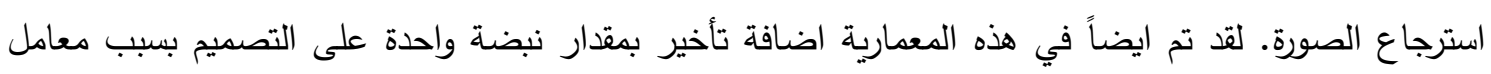

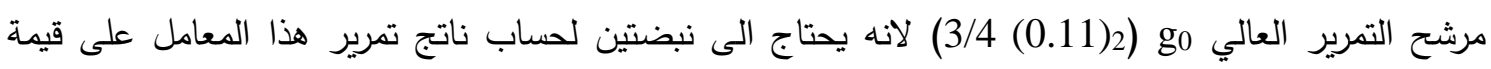

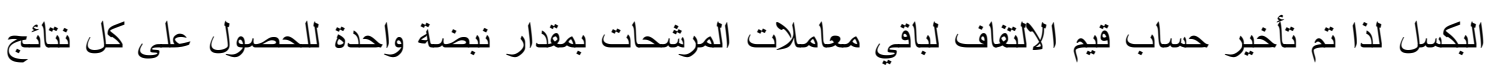
تمرير المعاملات على قيم البكسلات بنفس الوقت وبنفس النبضة لضمان الحصول على نتائج صحيحة. بعد اكمال

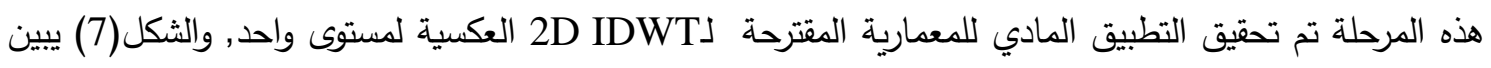

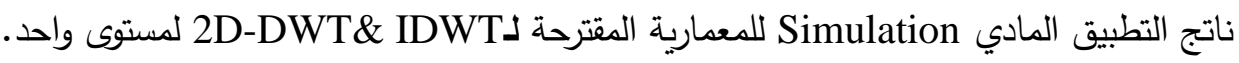

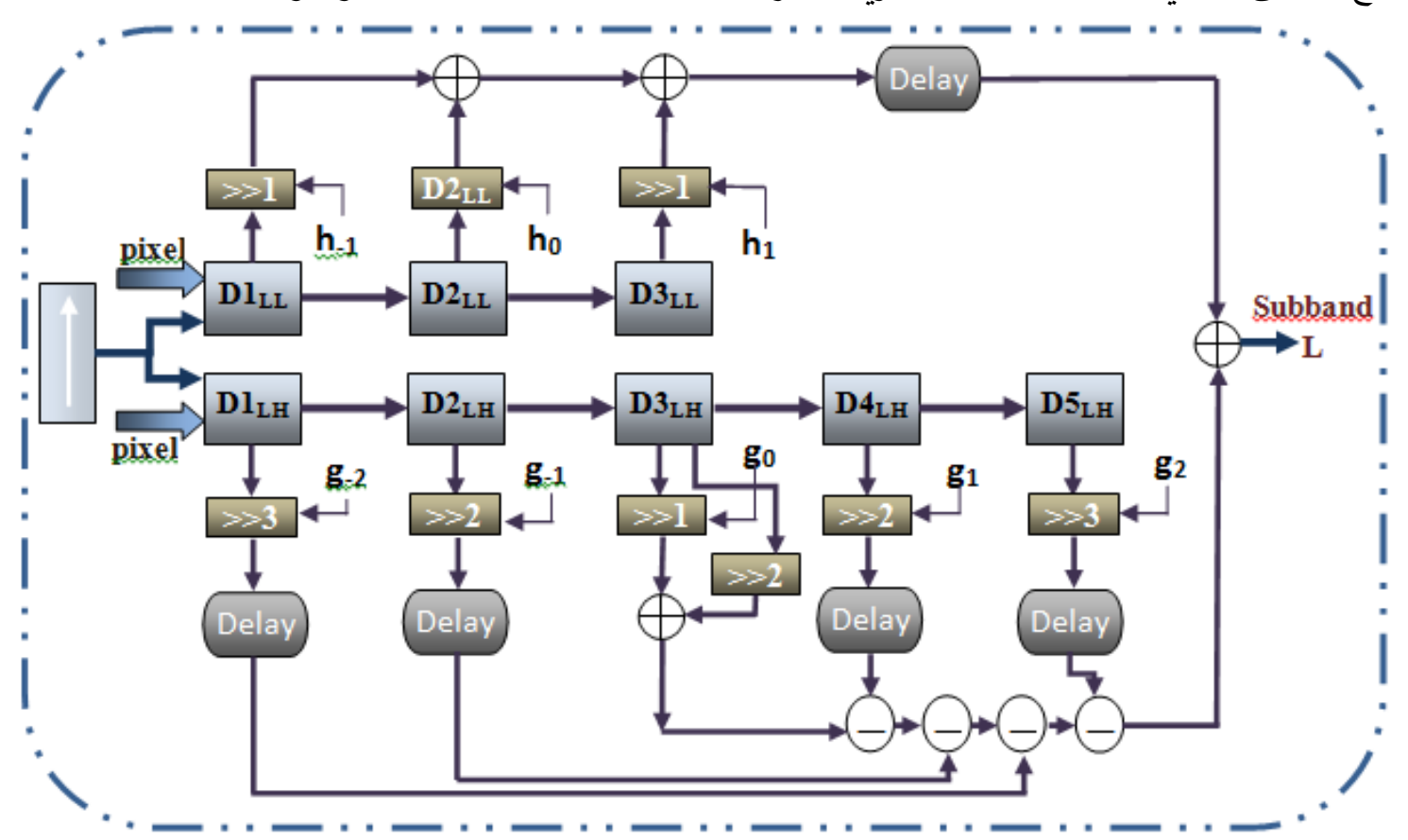

الثكل(6) الوصف المادي لعمل المعالج الاول (Process(DLL,DLH) 


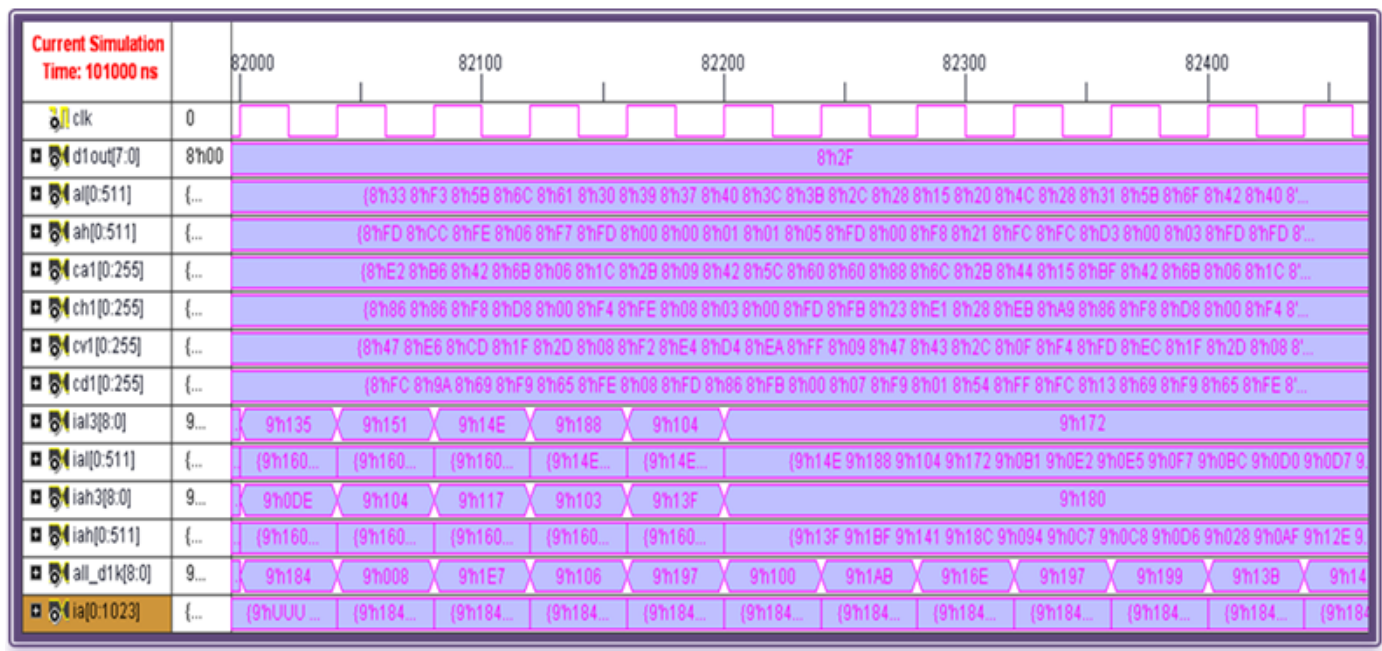

الثكل(7) نتائج Simulation للمعمارية المقترحة لـ 2D-DWT\& IDWT لمستوى واحد.

4. المعمارية المقترحة لخوارزمية 2D DWT-IDWT باعتماد معمارية القاسم لتطبيق الالتفاف الرياضي i التطبيق العملي للمعمارية المقترحة لـ 2D DWT الأمامية لمستوى واحد

لقد تم تصميم المعمارية لخوارزمية التحويل المويجي ذات البعدين ايضاً باعتماد طريقة القاسم لتطبيق الالتفاف الرياضي. ان التطبيق المادي للمعمارية المقترحة باعتماد القاسم لمستوى تحليل واحد مشابه للمعمارية

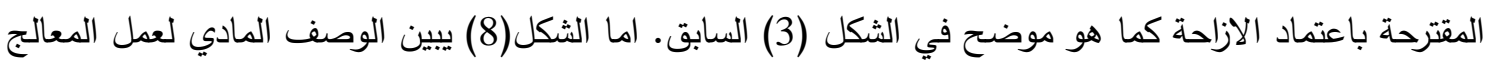
الاول في التحويل المويجة الامامي, حيث ان طريقة عمل هذا المعالج هي نفس طريقة المعالجة للمعالج الاول في لهي

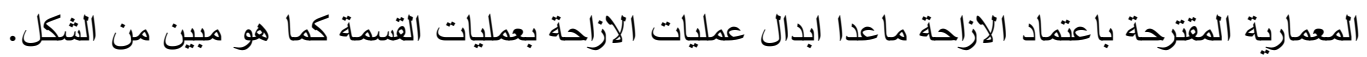

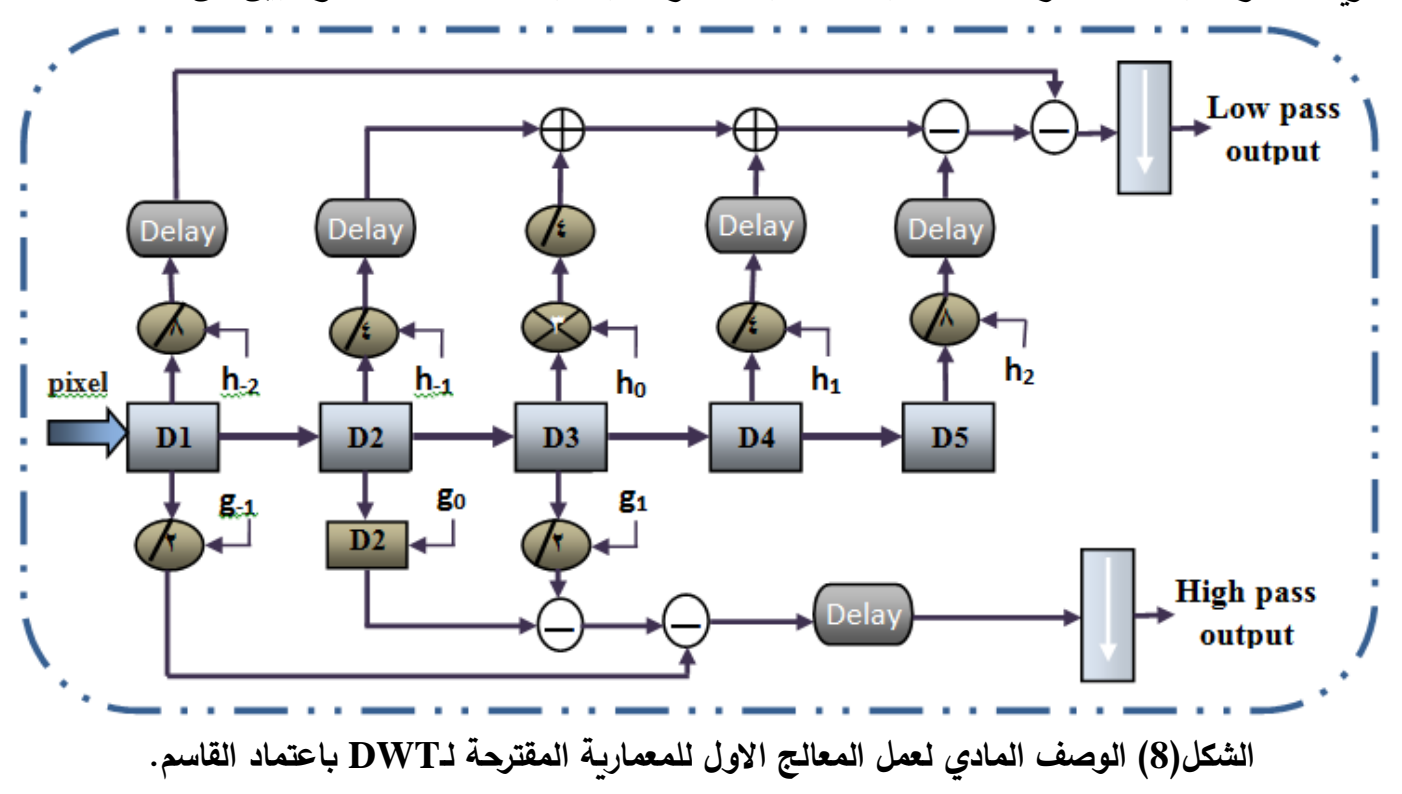

2D IDWT العكسية لمستوى واحد :ii التطبيق العملي للمعمارية المقترحة لـ

ان المعمارية العكسية المقترحة باعتماد القاسم مشابه للمعمارية المقترحة باعتماد الازاحة الموضحة في الثكل (5) السابق, كما ان طريقة عمل المعالجات في هذه المعمارية هي نفس طريقة العمل في المعمارية 
المقترحة باعتماد الازاحة ماعدا ابدال عمليات الازاحة بعمليات القسمة. الثكل(9) يبين الوصف المادي IDWT

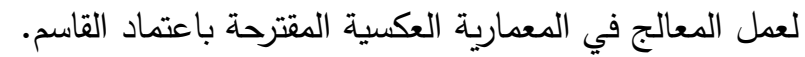

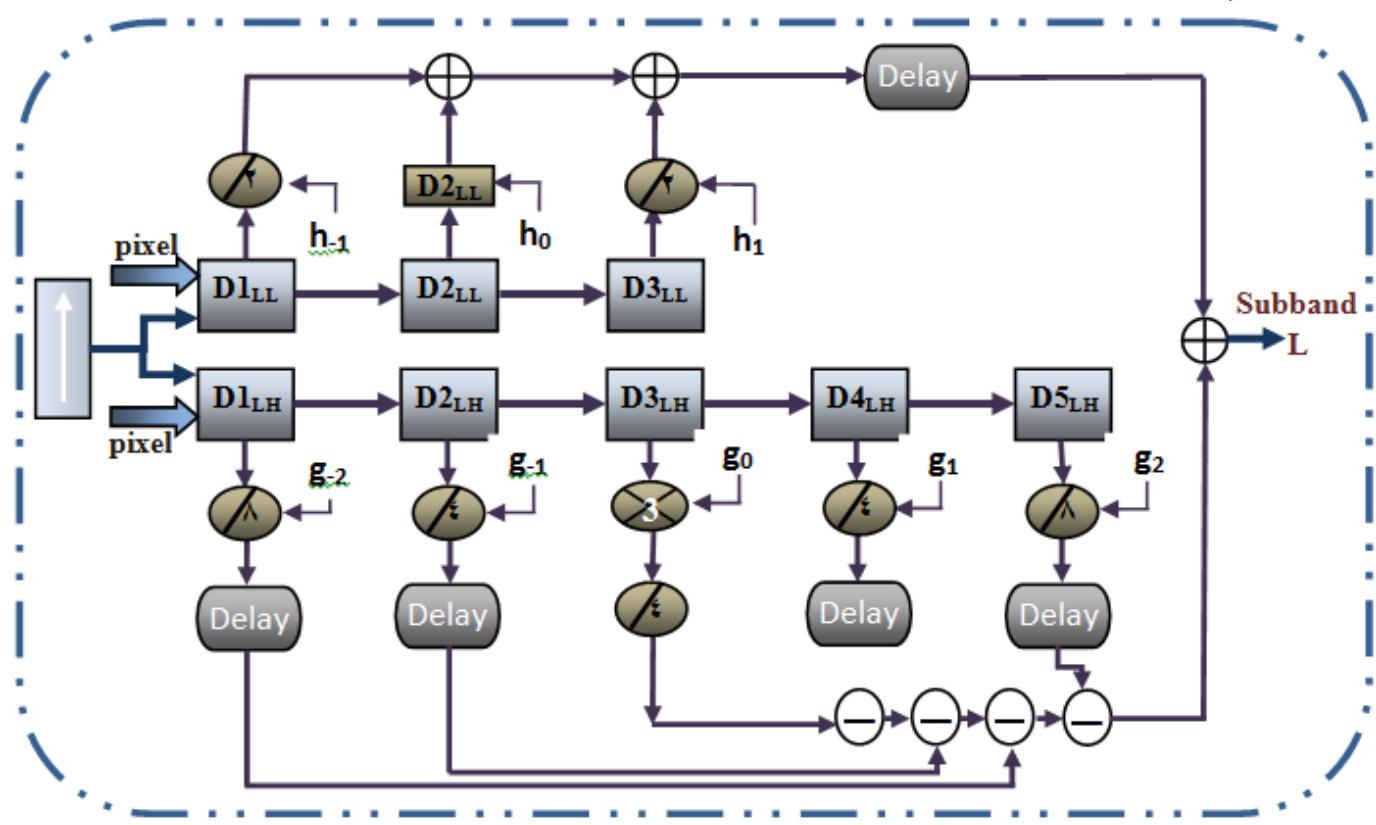

الثكل(9) الوصف المادي لعمل المعالج الاول للمعمارية المقترحة لـIDWT باعتماد القاسم.

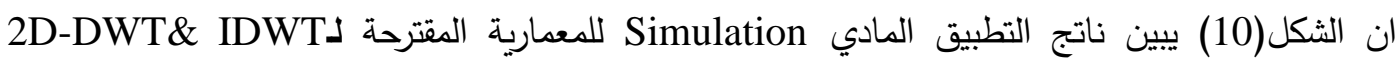
لمستوى واحد باعتماد طريقة القاسم لتطبيق الالتفاف.

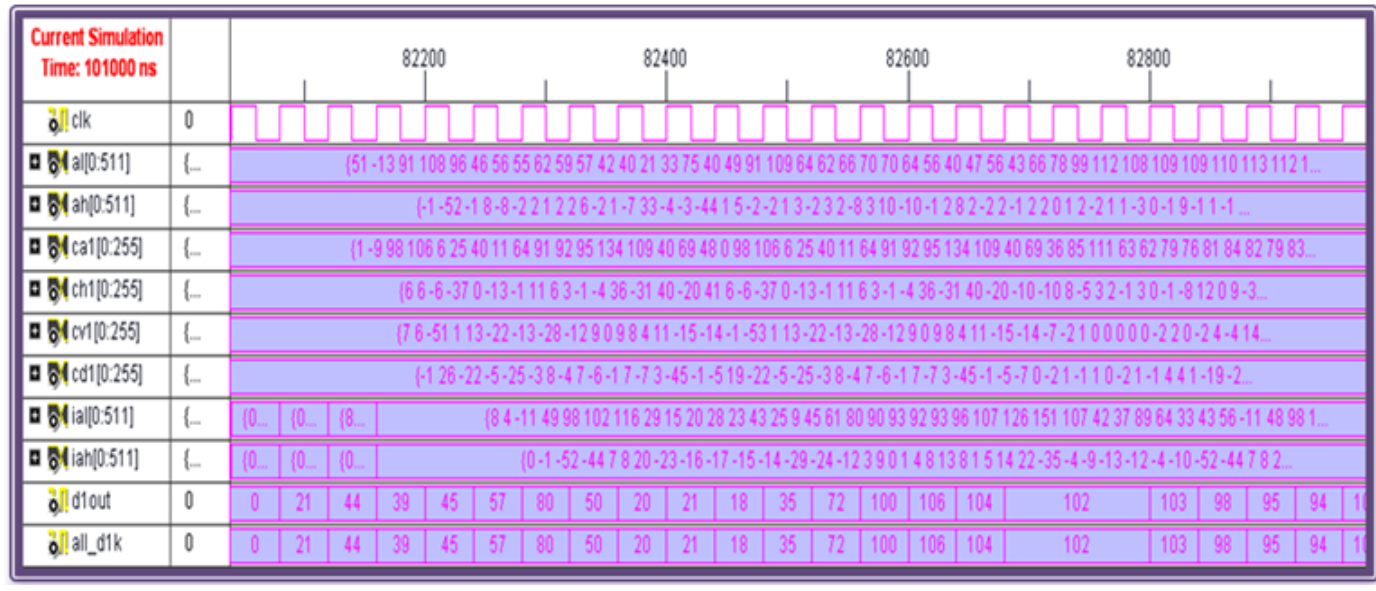

الثكل(10) نتائج Simulation للمعمارية المقترحة لـ2D-DWT\& IDWT لمستوى واحد باعتماد القاسم. ان الثكل (11) يوضح الصورة الأصلية والصور الثانوية لمستويين الناتجة من تتفيذ التحويل المويجي الأمامي وكذلك الصورة المسترجعة. أما الثكل (12) يبين عملية تحليل الصورة Boat لمستويين. 


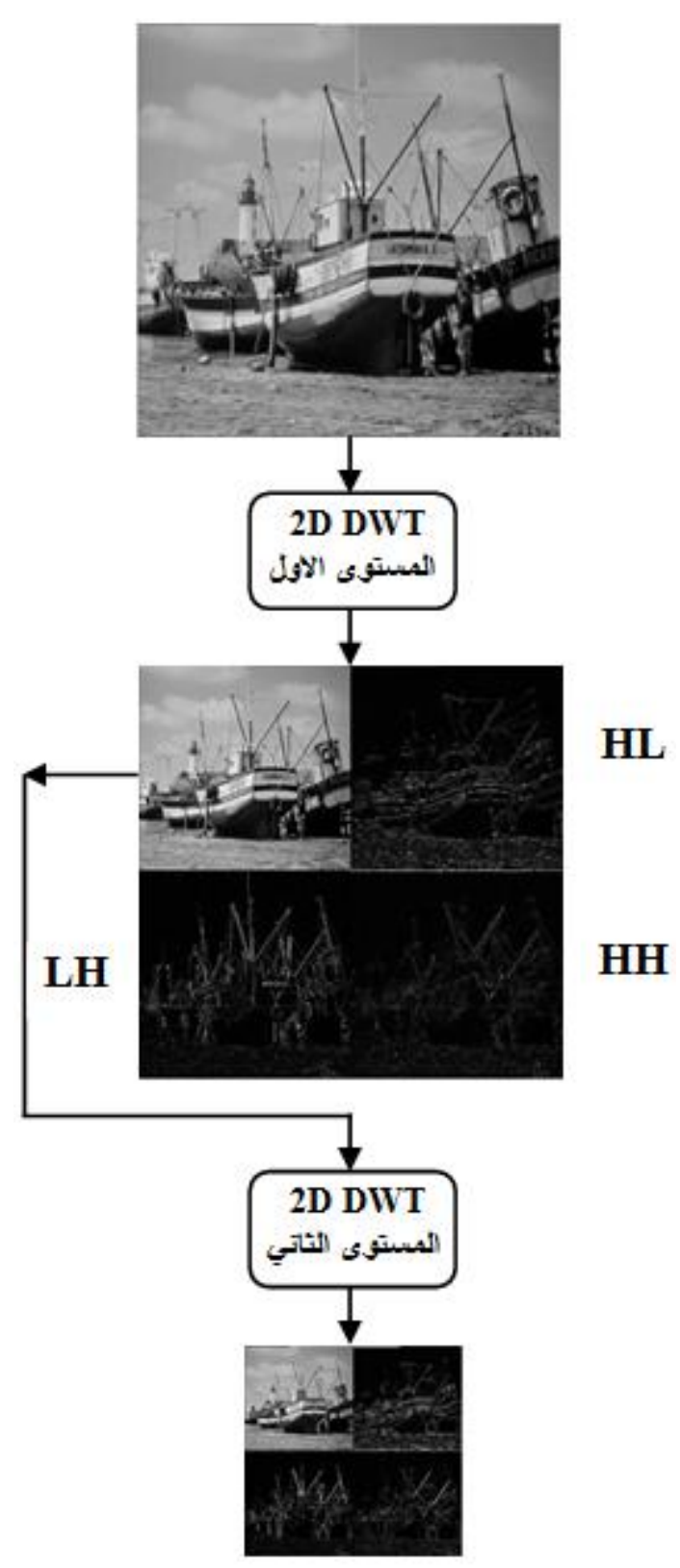

الثكل(11) تحليل الصورة Boat لمرشح 5/3 لمستويين. 

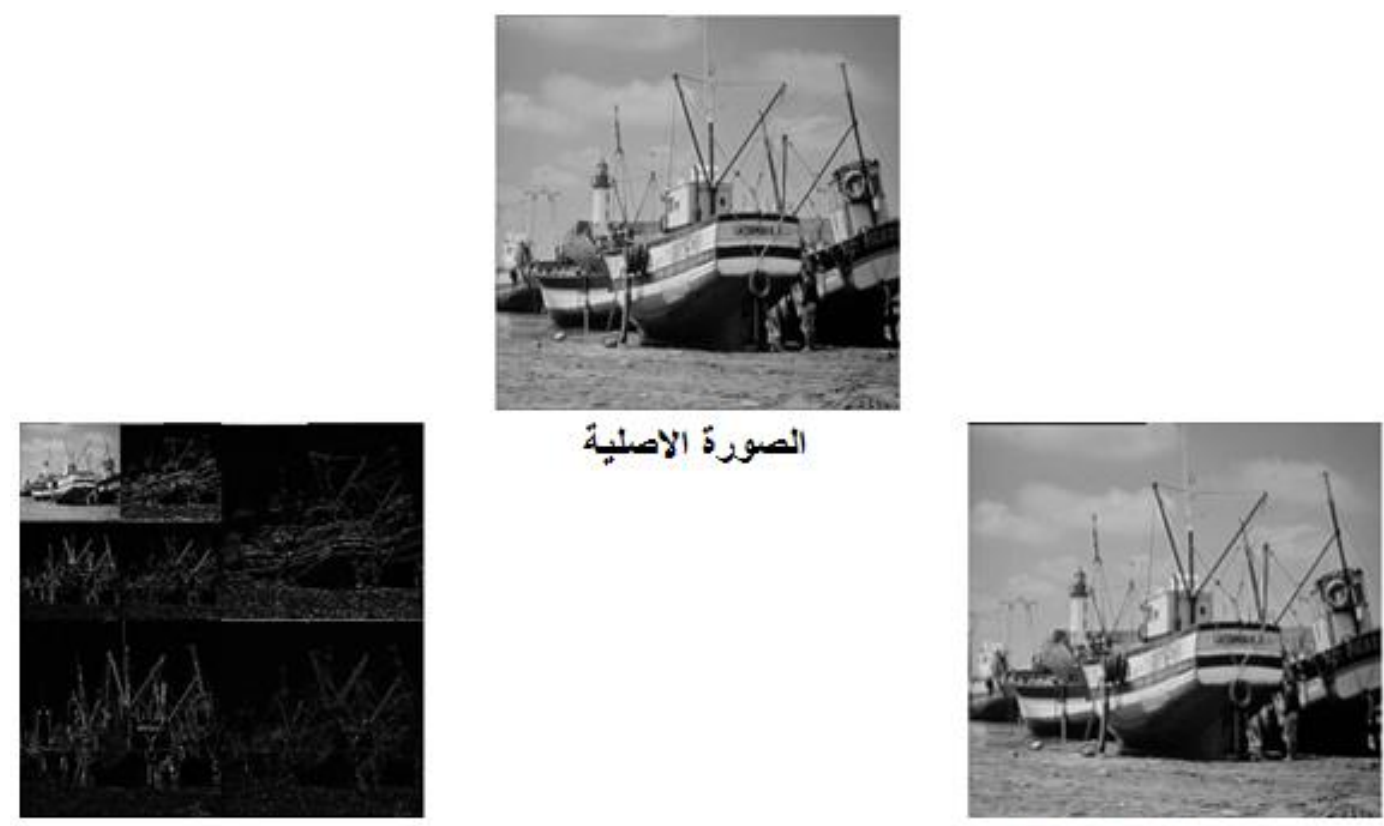

\section{الصورة المسترجعة}

الثكل(12) تحليل واسترجاع الصورة Boat لمرشح 5/3 .

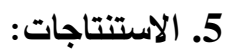

ان وجود عملية خط الانابيب ضمن المعماريات المقترحة تؤدي الى زيادة عنصر المعالجة عند كل نبضة,

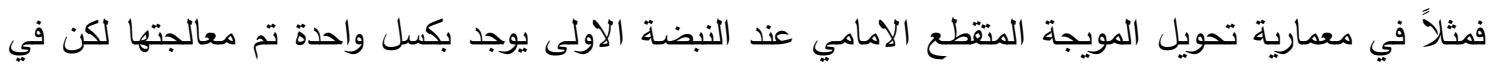
النبضة الثانية يزداد عدد البكسلات الى اثثين وهكذا يزداد العدد عند كل نبضة الى مابعد النبضة الخامسة حيث الثيل يستقر العدد الى خمسة بكسلات التي يتم معالجتها عند كل نبضة (اي ما بعد النبضة الخامسة), وهذا يعني زيادة

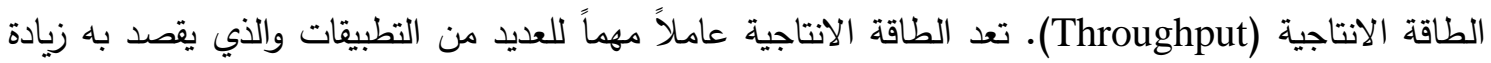
عدد البكسلات او العينات التي يتم معالجتها في وقت واحد وعند كل نبضة, وهذا يؤدي الى اداء افضل.كما ان وجود خاصية التوازي ضمن المعماريات المقترحة تؤدي الى تضاعف الطاقة الانتاجية ومن ثم زيادة عملية المعالجة عند كل نبضة, لذا ان وجود عملية خط الانابيب مع خاصية التوازي ضمن المعماريات المقترحة يؤدي الى معالجة كفوءة وأداء افضل. ان تطبيق المعمارية المقترحة لخوارزمية 2D DWT-IDWT عند اعتماد معمارية الازاحة في المعمارية المقترحة فإنه يستهلك عدد من الثرائح او الدوائر المنطقية من المنطقة ضمن شرائح (Slices) الجهاز , لكن عند

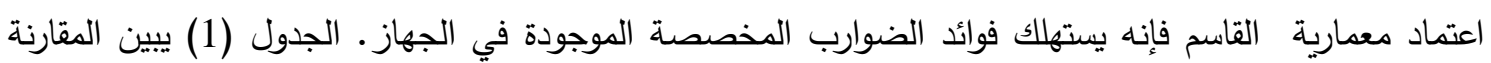
بين المعماريتين المقترحتين لخوارزمية التحويل المويجي باعتماد معمارية الازاحة والقاسم, تثير المقارنة ان عدد فئد الثرائح المستغلة تكون اقل عند اعتماد معمارية الازاحة والذي يؤدي الى تردد اعلى وزمن تتفيذ اقل. 
الجدول (1) المقارنة بين نتائج تطبيق المعماريتين المقترحتين لخوارزمية 2D-DWT\& IDWT باعتماد الازاحة والقاسم لمستوى واحد.

\begin{tabular}{|c|c|c|c|c|}
\hline نسبة الاستخدام & |العدد الكلي & العدد المستغل & المصادر & المعمارية المقترحة \\
\hline$\% 23$ & 4656 & 1085 & Slices & \multirow{7}{*}{ 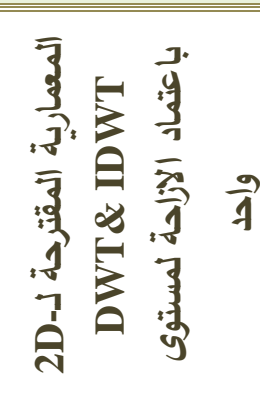 } \\
\hline$\% 12$ & 9312 & 1190 & Slice Flip Flops & \\
\hline$\% 20$ & 9312 & 1911 & 4 Input LUTs & \\
\hline$\% 3$ & 232 & 9 & Bonded IOBs & \\
\hline$\% 0$ & 20 & 0 & Multipliers & \\
\hline \multicolumn{3}{|c|}{11.18} & Min period & \\
\hline \multicolumn{3}{|c|}{89.445} & Max Frequency & \\
\hline$\% 25$ & 4656 & 1179 & Slices & \multirow{7}{*}{ 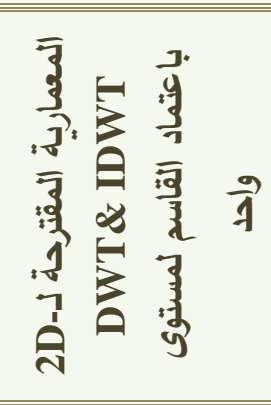 } \\
\hline$\% 12$ & 9312 & 1163 & Slice Flip Flops & \\
\hline$\% 20$ & 9312 & 1935 & 4 Input LUTs & \\
\hline$\% 14$ & 232 & 33 & Bonded IOBs & \\
\hline$\% 30$ & 20 & 6 & Multipliers & \\
\hline \multicolumn{3}{|c|}{$12.472 \mathrm{~ns}$} & Min period & \\
\hline \multicolumn{3}{|c|}{$80.180 \mathrm{MHz}$} & Max Frequency & \\
\hline
\end{tabular}




\section{|لمصادر}

[1] ISO/IEC FCD15444-1, (2000), "JPEG 2000 Image Coding System".

[2] ISO/IEC JTC1/SC29/WG11, FCD 14496-1, (1998), "Coding of Moving Pictures and Audio".

[3] Guoan Yang \& Huub Van de Wetering \& Songjun Zhang, (2012), "Optimization Design of Biorthogonal Wavelet Filter Banks for Extending JPEG 2000 Standard Part-2 ", J Sign Process Syst.

[4] Gaurav Tewari, Santu Sardar, K. A. Babu,(2011)," High-Speed \& Memory Efficient 2-D DWT on Xilinx Spartan3A DSP Using Scalable Polyphase Structure with DA for JPEG2000 Standard", IEEE,.

[5] D. U. Shah1, C. H. Vithlani2 , (2011),"Efficient Implementation of Discrete Wavelet Transforms Using FPGAs ", International Journal of Advances in Engineering \& Technology, Sept.

[6] Po-Cheng Wu and Liang-Gee Chen, (2001)," An Efficient Architecture for Two-Dimensional Discrete Wavelet Transform", IEEE Transactions On Circuits And Systems For Video Technology, Vol. 11, No. 4, April.

[7] Dhaha Dia, Medien Zeghid, Taoufik Saidani, Mohamed Atri, Belgacem Bouallegue,Mohsen Machhout and Rached Tourki,(2009),"Multi-level Discrete Wavelet Transform Architecture Design ", Proceedings of the World Congress on Engineering, Vol I, WCE 2009, July 1 - 3, London, U.K. , .

[8] M. Martina and G. Masera,(2007),"Multiplierless, Folded 9/7-5/3 Wavelet VLSI Architecture", IEEE Transactions on Circuits and Systems- II: Express Briefs, vol.54 No. 9, September.

[9] X. Chengyi, T. Jinwen and L. Jian ,(2006)," Low complexity reconfigurable architecture for the $5 / 3$ and $9 / 7$ discrete wavelet transform", Journal of Systems Engineering and Electronics vol. 17 No.2, pp. 303-308,. 